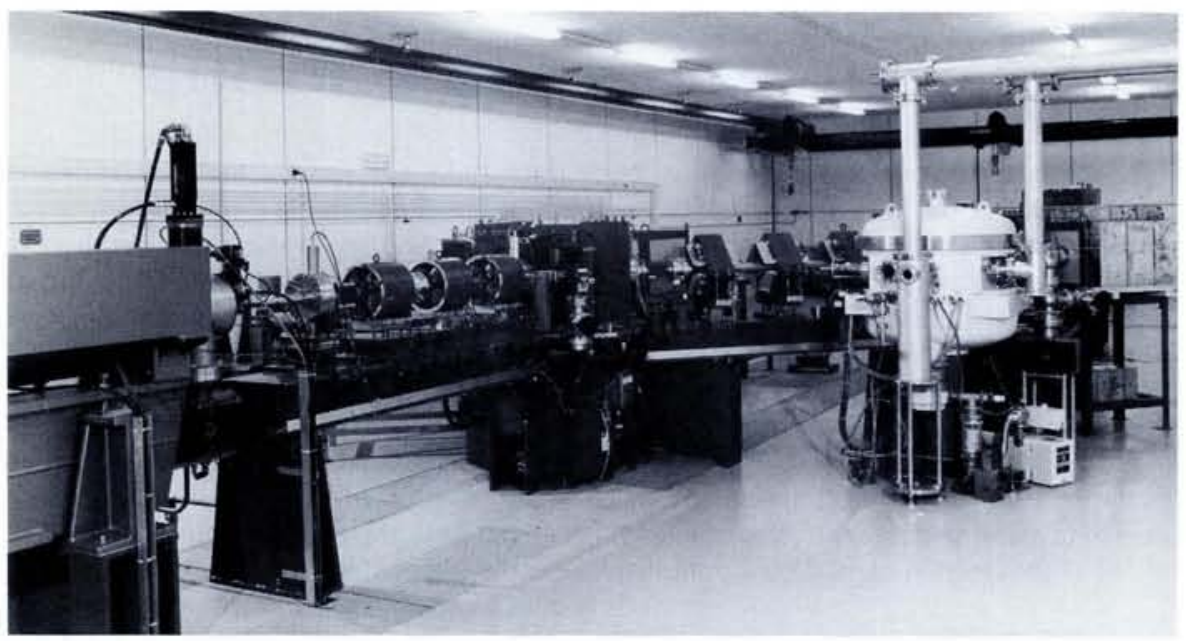

This linac-driven free-electron laser (FEL) at the FOM "Rijnhuizen" Institute for Plasma Physics, The Netherlands, became last year the first in Europe to produce radiation. Bending magnets and quadrupole lenses feed electrons from the linac (on the left) into a single-stage undulator/resonator structure hidden behind the vacuum tank housing one of the cavity mirrors. Infrared radiation with a wavelength of $2 \mu \mathrm{m}$ is produced at the $25 \mathrm{GeV}$ maximum energy. Storage rings are normally used instead of linacs for drivers at energies above $50-100 \mathrm{GeV}$ (wavelengths $\leq 500 \mathrm{~nm}$ to $1 \mu \mathrm{m}$ ). In a proposed [3] scheme for photo-ionisation injection for HIF, the idea is to use a conventional $215 \mathrm{MeV}$ linac-driven undulator/ resonator generating $240 \mathrm{~nm}$ radiation as the first stage of a two-stage device. This approach allows one to retain the most important advantage of a linac driver, namely the high brightness.

arrives at $N=4 \times 10^{15}$ ions. It is worthwhile to note, however, that a modified design allowing for more comfortable beam intensities $\left(N=10^{12}-10^{13}\right)$ is being studied at CERN.

\section{FEL photo-ionisation}

Photo-ionisation by a FEL appears possible because one would be operating in the UV region. What is needed is to work in the self-amplified spontaneous emission (SASE) regime. There are several alternatives for obtaining the $84 \mathrm{~nm}$ wavelength required for the $\mathrm{Bi}^{+} \rightarrow \mathrm{Bi}^{++}$photoionisation process starting with the 240 $\mathrm{nm}$ wavelength of a standard (e.g., $\mathrm{KrF}$ ) laser. One can use a frequency tripling device, and another possibility which is being explored is to employ an electron beam from a conventional linac of 200 $400 \mathrm{MeV}$ to obtain output UV parameters as follows: $80 \mathrm{~nm}$ wavelength, $3 \mathrm{MW}$ power, $40 \mathrm{~mm}$ rad emittance. The third harmonic of light emitted by a conventional magnetic undulator structure is amplified at a resonance phase in a second undulator [3] and a three-dimensional simulation of this process is already giving reliable results.

\section{Beam interaction and pellet design}

Beam-target interaction and the pellet design seem to be major issues needing further investigation, not only by computation and/or model calculations but also experimentally.

Depositing a specific power of $10^{15}$ $10^{16} \mathrm{~W} / \mathrm{cm}^{2}$ in an ICF target pellet to achieve ignition conditions poses serious problems. These are, of course, determined by fluid instabilities as well as by im- perfections in the target design and illumination symmetry. The consensus seems to favour indirect drive because of superior uniformity, provided the $\mathrm{X}$-ray conversion efficiency remains sufficient, which seems to be the case.

Target design and the corresponding physical issues are under study at the INFN, Frascati [5], taking into account the expertise acquired in the context of a laser fusion programme. The investigations have been and continue to be focussed on implosion symmetry and stability for both direct and indirect drive.
Inertial confinement fusion (ICF) is the only appropriate alternative to magnetically confined approaches. Original hopes centered on laser or light-ion drivers whereas there is now general agreement that heavy ions might provide a better solution. ICF offers several advantages, e.g., no magnetic coils, simple geometry of the reactor vessel, separation of driver and reactor vessel, and most important, no radioactive actinides. But these disadvantages have to be set against some drawbacks, e.g., sophisticated drivers, complex target technology linked with military applications (which is the reason why ICF is given great importance in the USA, where it is financed to a large
In the case of heavy-ion beam driven generators, preliminary analyses of thermal X-rays suitable for indirect drive have been performed and analytical models for evaluating the efficiency of radiators suitable for use in ICF target chambers are being developed [5].

\section{Perspectives}

The main elements of a future research programme should include:

1) Energy transfer modelling involving further studies (both theoretical and experimental) of the feasibility of ignition and of the amount of energy to be transferred from the beam to the fuel to achieve a net energy gain. Studies of phenomena arising in beam-target interaction using techniques based on either lasers (photon transfer into the target) and/or accelerators (uniformity, efficiency for ion to X-ray conversion) are essential.

2) Development of high-performance accelerators, where the design of non-Liouvillian stacking schemes including the photo-ionisation mechanism, i.e., the type of accelerating sequence, is the principle concern (as will be the case for the ItalianGerman collaboration).

It is the scientific community's hope that such a programme will be considered in the near future as an issue at the European level in working towards a new nuclear energy source.

[1] Badger B. et al., KIK-3202 (1981); KIK3840 (1985).

[2] Rubbia C., Nucl. Inst. \& Methods A278 (1988) 253.

[3] Bonifacio B. et al., Proc. AIP Conf. (AIP, New York, NY) 1984.

[4] Puglisi M. and Wrulich A., Workshop Report, Varenna (1989).

[5] Atzeni S., Europhys. Lett. 11 (1990) 639.

\title{
Make ICF Safe and Environmentally Acceptable
}

\author{
H. Schopper, a member of the Board chaired by Prof. U. Colombo \\ that evaluated Europe's fusion research in 1990, comments on the \\ importance of considering the environmental aspects of inertial con- \\ finement fusion.
}

extent from the defense budget), the activity induced in structures by fast neutrons, and finally the sizable tritium inventory.

So far, the world-wide effort both in inertial and magnetic confinement has concentrated on the physical and technical problems: these will eventually be solved. However, considering the history of fission reactors its seems urgently necessary to devote a comparable effort to safety and environmental problems (this is one of the major recommendations of the European Community's Evaluation Board). It is particularly important to demonstrate for ICF that the worst possible accident will present no major hazard to the population; that 
radioactive wastes from the operation of the plant will not require isolation from the environment for geological timespans; that the release of radioactivity and other wastes during normal operation and maintenance will be acceptable; that decommissioning and dismantling of the plant is feasible at acceptable cost and radiation exposures. These issue are as important as technical questions and they should be considered at a very early stage of future projects: indeed, they should be an integral part of their design concept. World-wide collaboration should also be directed at these tasks. Without such a policy it will be difficult to create the necessary acceptance for ICF among the general population.

\section{Antihydrogen as a Probe}

New particles tend eventually to be used as probes for something else so the once exotic positron is now widely used in condensed matter studies. For positrons, one generally measures the Doppler shift of the characteristic energy $(511 \mathrm{keV})$ of the two photons produced by the rapid (picosecond) annihilation of a positron by electrons. This shift is caused by changes in the positron's momentum due to trapping in crystalline defects (e.g., vacancies) or, as discovered recently, by temporary

\section{RESEARCH PROFESSORSHIP}

The Danish Natural Science Research Counsil (SNF) invites applications for a 5-year Research Professorship, associated the Mikroelektronik Centret at the Technical University of Denmark. The chair is financed by the development program in Material Sciences (MUP).

The successful applicant is selected by an expert commitee, established by SNF. Applications should be sent to SNF, H.C. Andersens Blvd. 40, DK-1553 Copenhagen V, Denmark before July 1. 1992. Applicants will receive the results of the final expert evaluation and they will not be published without the approval of the applicants.

The research professor shall strenghen MIC's R\&D program within advanced semiconductor materials and their applications in optoelectronic components and devices. In particular, nonlinear ultra-fast dynamics is of interest aiming at optical switching. The successful candidate is therefore expected to document broad experience in ps and fs spectroscopy as well as a profound knowledge of modern lowdimensional semiconductor materials.

Mikrolektronik Centret is responsible for training engineering students in semiconductor component physics and process technology. The research professor is responsible for supervising PH.D. students and give advanced graduate lectures.

In addition to excellent professional skills, the staff at MIC is expected to contribute to the dynamical research environment rapidly being established. We expect flexibility and ability to redirect our research towards rewarding competitive goals. Further information can be obtained from Director Ove Poulsen, tel. +4545931222.5744 or from SNF, tel. +4533154645.' 210.

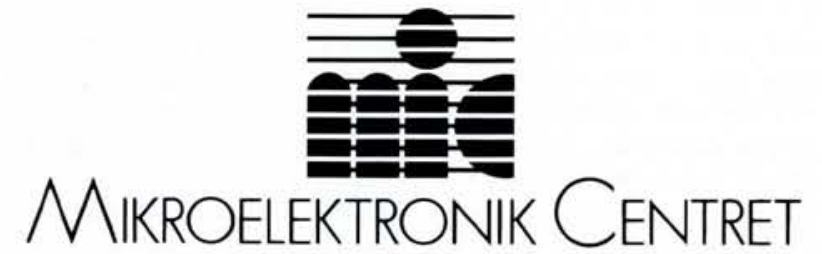

DTH, DK-2800 Lyngby, Denmark

MIC is a new autonomous research center, established at the Technical University of Denmark. Its research mission is within semiconductor physics and technology and semiconductor based micro-technologies.

Major Danish industries and universities work together at MIC. Our new process laboratory will be operational in the summer of 1993. It will provide

unique possibilities for synthesizing and processing advanced materials.

potential wells formed by fluctuations in conducting liquids.

The antiproton may now become a probe since a University of Tokyo group showed last year [lwasaki M., et al., Phys. Rev. Lett. 67 (1991) 1246] that $3.6 \%$ of the antiprotons fired into liquid $\mathrm{He}$ were trapped for up to $30 \mathrm{~ms}$ whereas the remainder were annihilated some $10^{8}$ times faster (within a few ps, the theoretically predicted time). As long ago as 1964 , G.T. Condo suggested that the quantum numbers of some exotic $\mathrm{He}$ atoms formed by replacing one of the electrons in Bohr-like orbits around the nucleus with an antiproton does not mean that the remaining electron is ejected immediately (as assumed by others in calculating the antiproton lifetime). The Pauli exclusion principle would then disallow the collisions by surrounding atoms which bring about a short-circuiting of the antiproton's slow cascade through a series of atomic states - a cascade that ends with the antiproton being within range of the strong interaction causing annihilation. So it is thought that a few percent of the $\mathrm{He}$ atoms are special in some fascinating way.

A joint experiment PS205 (involving Tokyo University, MPI für Quantenoptik and CERN) at CERN's LEAR accelerator which produces the world's cleanest, monoenergetic, low energy antiproton beam of small spot size has reproduced the effect. Further work is in progress using the various forms of He to identify what accounts for the stability of the long-lived metastable $\mathrm{He}$ atoms (the "trapping states"), to look for light emission, and to laser pump the metastable atoms to prolong their lifetimes even further to allow precision spectroscopy. Reacting metastable atoms with positrons and positronium may produce antihydrogen - which could be used as a physics "laboratory" ... and as a probe.

\section{Antihydrogen Workshop}

30 - 31 July 1992

Ludwig-Maximilians-Universität, Munich, Germany

Organised by:

CERN and MPI für Quantenoptik

Topics: why antihydrogen?; ultra-low energy antiprotons; positron accumulation \& positronium; routes to antihydrogen production; antihydrogen: trapping, spectroscopy \& laser simulated recombination.

\section{Contact:}

C. Zimmermann, MPI für Quantenoptik, Postfach 15413, W-8046 Garching (Fax: +49-89-32 9552 00) 